\title{
Boundary Conditions in Case of Spatial Resonance Dispersion
}

\section{Skettrup, Torben; Balslev, I.}

\section{Published in:}

Physical Review B Condensed Matter

Link to article, DOI:

10.1103/PhysRevB.3.1457

Publication date:

1971

\section{Document Version}

Publisher's PDF, also known as Version of record

Link back to DTU Orbit

\section{Citation (APA):}

Skettrup, T., \& Balslev, I. (1971). Boundary Conditions in Case of Spatial Resonance Dispersion. Physical Review B Condensed Matter, 3(4), 1457-1462. https://doi.org/10.1103/PhysRevB.3.1457

\section{General rights}

Copyright and moral rights for the publications made accessible in the public portal are retained by the authors and/or other copyright owners and it is a condition of accessing publications that users recognise and abide by the legal requirements associated with these rights.

- Users may download and print one copy of any publication from the public portal for the purpose of private study or research.

- You may not further distribute the material or use it for any profit-making activity or commercial gain

- You may freely distribute the URL identifying the publication in the public portal

If you believe that this document breaches copyright please contact us providing details, and we will remove access to the work immediately and investigate your claim. 
${ }^{28}$ See, e.g., C. K. Chau, M. V. Klein, and B. Wedding, Phys. Rev. Letters 17, 521 (1966).

${ }^{29}$ R. J. Rollefson, $\mathrm{Ph}$. D. dissertation, Cornell University, 1970 (unpublished)。

${ }^{30}$ Indirect evidence for the purity of the best of these samples is provided by the work on the effects of impurities on color center formation by M. Meistrich, Ph. D. dissertation, Cornell University, 1967 (unpublished).

${ }^{31}$ C. T. Walker, Phys. Rev。132, 1963 (1963).

${ }^{32}$ We are indebted to $R$ 。 K. Skogerboe and the Analytical Laboratory of the Cornell University Materials Science Center for these analyses.

${ }^{33} \mathrm{High}$ critical temperature of $\mathrm{Pb}$ permits a quite wide range of operation in the superconducting region, and the pure material was chosen in order to achieve a reasonable sensitivity above the transition temperature. J. K. Wigmore [Appl. Phys. Letters $\underline{13}, 73$ (1968)] has reported an interesting bolometer based on the avalanche phenomenon in a doped semiconductor for use at low temperatures in strong magnetic fields.

${ }^{34}$ C. P. Enz, Phys. Letters 20,442 (1966).

${ }^{35}$ A. Griffin, Phys. Letters 17,208 (1965).

${ }^{36}$ P. C. Kwok and P. C. Martin, Phys. Rev. 142, 495 (1966).

${ }^{37}$ R. J. Hardy, Phys. Rev. B 2 , 1193 (1970).

${ }^{38}$ For a calculation starting from the Boltzmann transport equation see P. Erdos and S. B. Haley, Phys. Rev. 184, 951 (1969).

${ }^{39}$ See, e.g., L. D. Landau and E. M. Lifshitz, Fluid Mechanics (Pergamon, Oxford, England, 1959), p. 49.

${ }^{40} \mathrm{M}$. Chester, Phys. Rev. 131, 2013 (1963).

${ }^{41}$ See Ref. 39, p. 307.

${ }^{42}$ The relationship between the phase and energy velocity of acoustic waves in cubic crystals has been discussed in detail by G. F. Miller and M. J. P. Musgrave, Proc. Roy. Soc. (London) A236, 352 (1956).

${ }^{43}$ R. N. Claytor and B. J. Marshall, Phys. Rev. $\underline{120}$, 332 (1960).

${ }^{44}$ C. V. Briscoe and C. F. Squire, Phys. Rev. $\underline{106}$, 1175 (1957).
${ }^{45} \mathrm{~J}$. Vallin, K. Marklund, J. O. Sikström, and O. Beckman, Arkiv Fysik 32, 515 (1966).

${ }^{46} \mathrm{~B}$. Taylor, H. J. Maris, and C. Elbaum, Phys. Rev. Letters $\underline{23}, 416$ (1969).

${ }^{47}$ Transport of heat across the heater crystal interface has been considered by R. J. von Gutfeld, A. H. Nethercot, Jr., and J. A. Armstrong, Phys. Rev. 142, 436 (1966).

${ }^{48}$ R. C. Hanson, J. Phys. Chem. Solids 28,475 (1967).

${ }^{49}$ J. C. F. Brock, Ph. D。 dissertation, Oxford University, Oxford, England, 1965 (unpublished).

${ }^{50}$ From geometric considerations H. G. B. Casimir calculated that, for a long crystal of square cross section with side $d$, the mean free path $l=1.12 d$ [Physica $\underline{5}, 495$ (1938)].

${ }^{51}$ M. G. Holland, J. Appl. Phys. $\underline{33}, 2910$ (1962).

${ }^{52}$ D. D. Betts, A。B。 Bhatia, and $\bar{M}$ 。 Wyman, Phys . Rev. 104, 37 (1956).

${ }^{53}$ For a full discussion of the problem, reference should be made to Appendix E of Ref. 12。

${ }^{54}$ W. J. Parker, R. J. Jenkins, C. P. Butler, and G. L. Abbott, J. Appl. Phys. 32, 1679 (1961).

${ }^{55}$ This term has been used successfully in the analysis of heat-pulse propagation in liquid He II at low temperatures [J. M. Ziman, Phil. Mag. 45, 100 (1954)].

${ }^{56}$ See, e.g., H. S. Carslaw and J. C. Jaeger, Conduction of Heat in Solids, 2nd ed. (Oxford U. $\mathrm{P}_{\circ}$, London, 1959), p. 260 .

${ }^{57}$ C. Herring, Phys. Rev. 95, 954 (1954)。

${ }^{58}$ P. C. Kwok and P. B. Miller, Phys. Rev. 146, 592 (1966)。

${ }^{59}$ Changes in conductivity which were determined from the heat-pulse measurements were in reasonable agreement with Walker's data (see Ref. 32) for similarly irradiated $\mathrm{NaF}$.

${ }^{60}$ This conclusion has recently received further support from work on still purer NaF crystals [H. E. Jackson, C. T. Walker, and T. F. McNelly, Phys. Rev. Letters $\underline{25}, 26(1970)]$.

\title{
Boundary Conditions in Case of Spatial Resonance Dispersion
}

\author{
T. Skettrup \\ Physics Laboratory III, Technical University of Denmark, Lyngby, Denmark \\ and \\ I. Balslev \\ Physics Institute, Odense University, Odense, Denmark
}

(Received 21 September 1970)

\begin{abstract}
A simple model accounting for the influence of a surface on polariton waves is introduced. From this the extra boundary condition needed in case of a dispersive resonance is derived. The general result is discussed in relation to actual resonances in crystals. The theory of bulk waves and their boundary conditions is extended to include two or more dispersive reso-' nances. The behavior of the measured exciton lines in $\mathrm{ZnO}$ agrees well with the computed spectra.
\end{abstract}

\section{INTRODUCTION}

Spatial resonance dispersion ${ }^{1,2}$ may be important whenever crystalline excitations that couple to light are able to move. One of the characteristic features of this phenomenon is that two eigenmodes 
are excited by external monochromatic light. The presence of additional modes implies that extra boundary conditions are necessary in order to calculate reflection and transmission spectra.

The problem of the extra boundary condition needed in the case of a single resonance exhibiting dispersion has been discussed by several authors. ${ }^{1-5}$ Theories due to Pekar ${ }^{1}$ and to Hopfield and Thomas ${ }^{2}$ prescribe that the polarization associated with the resonance vanishes at ${ }^{1}$ or near ${ }^{2}$ the surface in case of normal incidence. This condition seems to be generally accepted by now. However, the microscopic surface properties leading to this condition have not been discussed in detail in previous papers. For example, it is not clear how strong the surface distortion should be for a sufficient quenching of the surface polarization. Furthermore, the above condition is insufficient in the case of several resonances, each requiring an extra boundary condition.

The purpose of this paper is to clarify these points and to discuss the boundary-condition problem on basis of a simple model of a crystal boundary. The model involves a single surface layer with specific resonance properties and thickness. This treatment leads to a clear distinction between situations where the polarization $P$ or the derivative $\partial P / \partial x$ vanishes at the surface. The application of our results to actual resonances in crystals leads to the accepted condition that $P=0$ at the surface. We have derived appropriate boundary conditions in case of two or more closely lying resonances and applied the results to the exciton spectrum of $\mathrm{ZnO}$.

\section{BULK POLARITON MODES}

Let us consider a one-dimensional crystal with a transverse undamped resonance exhibiting dispersion. In the absence of coupling to light the crystalline excitations associated with this resonance have eigen frequencies given by

$$
\omega=\omega_{0}+\hbar k^{2} / 2 M,
$$

where $\omega_{0}$ is the zero-wave-vector resonance frequency, and $M$ is the mass characterizing the dispersion. The coupled differential equations describing the interaction between electromagnetic and crystalline waves may be expressed as

$$
\begin{aligned}
& \frac{\partial^{2} A}{\partial t^{2}}-\frac{c^{2}}{\epsilon_{\infty}} \frac{\partial^{2} A}{\partial x^{2}}=\frac{1}{\epsilon_{0} \epsilon_{\infty}} \frac{\partial P}{\partial t}, \\
& \frac{\partial^{2} P}{\partial t^{2}}-\frac{\hbar \omega_{0}}{M} \frac{\partial^{2} P}{\partial x^{2}}+\omega_{0}{ }^{2} P=-\epsilon_{0} F \frac{\partial A}{\partial t} .
\end{aligned}
$$

Here $P$ is the polarization associated with the resonance, $A$ is the vector potential, $\epsilon_{\infty}$ is the background dielectric constant, $\epsilon_{0}$ and $c$ are permittivity and light velocity of vacuum, respectively, and
$F / \omega_{0}^{2}$ is the zero-frequency polarizability due to the resonance. Equation (2) is derived from Maxwell's equations, and Eq. (3) is the equation of motion for the polarization. Thus, without coupling $(F=0)$ Eq. (3) yields the dispersion of Eq. (1) if we neglect $k^{4}$ terms. In the absence of the second term of Eq. (3) we are dealing with local response of the medium. ${ }^{6}$

The polariton waves propagating in the crystal are found from the eigenmodes of the coupled equations (2) and (3). Hence the polariton dispersion can be expressed $\mathrm{as}^{2}$

$$
k^{2} c^{2} / \omega^{2}=\epsilon_{\infty}+F /\left(\omega_{0}^{2}-\omega^{2}+\hbar \omega_{0} k^{2} / M\right) .
$$

For a fixed (real) frequency this equation has two (possibly complex) solutions as shown in Fig. 1. Each mode is characterized by quantities $k_{i}, P_{i}$, and $A_{i}(i=1,2)$ related by

$P_{i}=-i \omega \epsilon_{0}\left[\left(k_{i}^{2} c^{2} / \omega^{2}\right)-\epsilon_{\infty}\right] A_{i}=-i \omega_{0} \epsilon_{0}\left(n_{i}^{2}-\epsilon_{\infty}\right) A_{i}$,

where $n_{i}$ is the refractive index.

\section{POLARITON MODES AT CRYSTAL BOUNDARY}

We shall discuss the influence of a surface on the basis of a transformation of the differential equations into difference equations. Let the crystal be divided into cells (see Fig. 2) defined by the width $a$, which must be greater than or equal to the lattice distance. In the long-wave limit $(k a \ll 1)$ the spatial second-order derivatives may be replaced by second-order differences. Equations (2) and (3) are
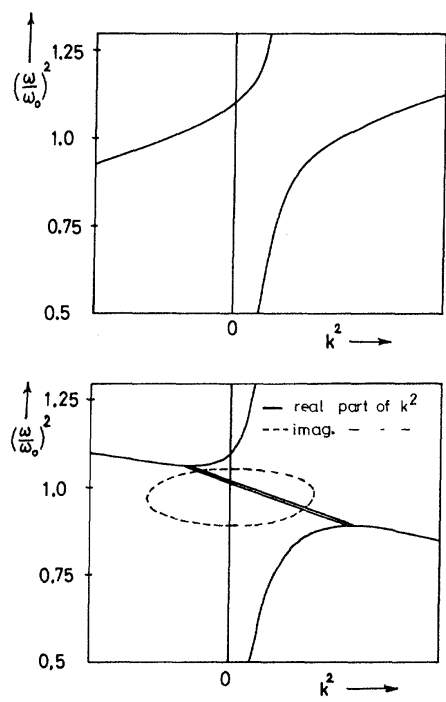

FIG. 1. Polariton dispersion curves shown as a $k^{2}-\omega^{2}$ dependence. The upper part corresponds to positive mass dispersion of the crystalline excitation, the lower part to negative mass. In the latter case, there is a frequency region where $k^{2}$ is complex with the imaginary part shown as a dotted curve. 

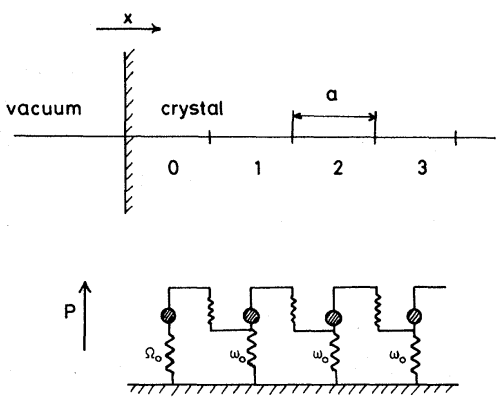

FIG. 2. Model of a one-dimensional crystal with a single dispersive resonance. In the mechanical analog, the lower springs have resonance frequencies as indicated. The upper springs represent nonlocal effects responsible for the dispersion.

equivalent to

$$
\begin{aligned}
\frac{\partial^{2} A^{n}}{\partial t^{2}} & -\frac{c^{2}}{\epsilon_{\infty} a^{2}}\left(A^{n-1}-A^{n}\right) \\
& -\frac{c^{2}}{\epsilon_{\infty} a^{2}}\left(A^{n+1}-A^{n}\right)=\frac{1}{\epsilon_{0} \epsilon_{\infty}} \frac{\partial P^{n}}{\partial t}, \\
\frac{\partial^{2} P^{n}}{\partial t^{2}} & -\frac{\hbar \omega_{0}}{M a^{2}}\left(P^{n-1}-P^{n}\right) \\
& -\frac{\hbar \omega_{0}}{M a^{2}}\left(P^{n+1}-P^{n}\right)+\omega_{0}^{2} P^{n}=-\epsilon_{0} F \frac{\partial A^{n}}{\partial t},
\end{aligned}
$$

where $A^{n}$ and $P^{n}$ are the vector potential and polarization in the $n$th cell. The second and third term of Eq. (7) represent "nonlocal forces" on the polarization. For example, the microscopic mechanisms responsible for the second term may be interpreted as a nearest-neighbor interaction between cell No. $n-1$ and cell No. $n$. This is visualized in the mechanical analogue in Fig. 2. Let the outermost cell in a semiinfinite crystal be cell No. 0 . Then Eqs. (6) and (7) are valid for $n \geqq 1$. An incoming wave from the left (vacuum) excites three modes, namely, a reflected wave and two right-running polariton waves. It is easily shown that the amplitudes of these waves are established from Maxwell's boundary conditions (continuity of $A$ and $\partial A / \partial x)$ and the equation of motion for the polarization in cell No. 0 . This equation of motion therefore provides the extra boundary condition needed in this model.

At this point we want to specify our model of a boundary. First, it is reasonable that the second term in Eq. (7) vanishes for $n=0$. There is simply no "nonlocal force," on the polarization from vacuum. Second, we shall account for a distortion of the resonance in a surface layer by assuming that cell No. 0 has a resonance frequency $\Omega_{0}$ different from that of the bulk crystal. On the other hand, we specifically assume that all other cells $(n \geqq 1)$ are perfectly tuned to the common frequency $\omega_{0}$. Thus, in our model the length $a$ should be chosen to be the width of the surface layer with a resonance frequency considerably different from $\omega_{0}$. How ever, for relevant polariton modes we still assume that $k a \ll 1$.

According to our model, the equation of motion for cell No. 0 is

$$
\left(\Omega_{0}^{2}-\omega^{2}\right) P^{0}-\frac{\hbar \omega_{0}}{M a}\left(\frac{\partial P}{\partial x}\right)^{0}=i \omega \epsilon_{0} F A^{0},
$$

where we have inserted a harmonic time variation given by $\omega$ and used

$$
P^{1}-P^{0} \approx a\left(\frac{\partial P}{\partial x}\right)^{0} .
$$

Splitting up the polarization and vector potential of the 0th cell into contributions from the uncoupled polaritons [Eq. (5)], we obtain

$$
\begin{gathered}
P^{0}=P_{1}^{0}+P_{2}^{0}, \\
\left(\frac{\partial P}{\partial x}\right)^{0}=i k_{1} P_{1}^{0}+i k_{2} P_{2}^{0},
\end{gathered}
$$

and

$$
A^{0}=A_{1}^{0}+A_{2}^{0} .
$$

From Eqs. (4), (5), and (8)-(11), we find

$$
\begin{aligned}
& P_{1}{ }^{0}\left[\Omega_{0}^{2}-\omega_{0}^{2}-\left(\hbar \omega_{0} / M\right) k_{1}^{2}-i\left(\hbar \omega_{0} / M a\right) k_{1}\right] \\
& \quad+P_{2}^{0}\left[\Omega_{0}^{2}-\omega_{0}^{2}-\left(\hbar \omega_{0} / M\right) k_{2}^{2}-i\left(\hbar \omega_{0} / M a\right) k_{2}\right]=0 .
\end{aligned}
$$

The third term in each bracket is ignorable when compared to the fourth term since $k a \ll 1$. Then

$$
\frac{P_{2}^{0}}{P_{1}^{0}}=-\frac{\Omega_{0}^{2}-\omega_{0}^{2}-i k_{1} \hbar \omega_{0} / M a}{\Omega_{0}^{2}-\omega_{0}^{2}-i k_{2} \hbar \omega_{0} / M a} .
$$

It is convenient in the further discussion to introduce the quantity $d$ describing the "relative detuning" of the surface layer by the definition

$$
d=\left(\Omega_{0}^{2}-\omega_{0}^{2}\right) / \omega_{0} \omega,
$$

and the "Compton wavelength"

$$
\lambda_{M}=h / M c
$$

associated with the dispersion. Then

$$
\frac{P_{2}^{0}}{P_{1}^{0}}=-\frac{1-i n_{1} \lambda_{M} /(2 \pi a d)}{1-i n_{2} \lambda_{M} /(2 \pi a d)}
$$

or

$$
P^{0}-\frac{c \lambda_{M}}{2 \pi a d \omega}\left(\frac{\partial P}{\partial x}\right)^{0}=0 .
$$

The general boundary condition derived from this model then requires that a linear combination of $P$ and $(\partial P / \partial x)$ should vanish at the surface. This is consistent with the result of Deutsche and $\mathrm{Mead}^{5}$ 
stating that only this type of boundary conditions is allowed in a causal system.

We are now able to distinguish between two extreme types of surface effects:

(a) Large detuning $(d \approx 1)$ in a surface layer considerably larger than $\lambda_{M}$, such that

$$
2 \pi a d / \lambda_{M} \gg\left|n_{1}\right| \text { and }\left|n_{2}\right| \text {. }
$$

Then

$$
P_{1}^{0}+P_{2}^{0}=P^{0} \approx 0,
$$

which is the boundary condition reported in Refs. 1 and 2.

(b) Small detuning $(d \ll 1)$ and/or small width of the surface layer, such that

$$
2 \pi a d / \lambda_{M} \ll\left|n_{1}\right| \text { and }\left|n_{2}\right| \text {. }
$$

Then

$$
n_{1} P_{1}^{0}+n_{2} P_{2}^{0} \approx 0
$$

or

$$
\left(\frac{\partial P}{\partial x}\right)^{0} \approx 0
$$

\section{APPLICATION TO PHYSICAL CRYSTALS}

In this section we shall insert appropriate magnitudes for $M, a$, and $d$ in the condition derived in Sec. III. The dispersions of crystalline excitations encountered in nature are usually characterized by a magnitude of the mass $M$ of the order of, or larger than, the free-electron mass. This holds for free excitons in II-VI compounds and for optical lattice vibrations in ionic crystals (in the latter case $M<0$ ). Thus $\lambda_{M}$ is smaller than $10^{-2} \AA$. The smallest meaningful width of a surface layer is the interatomic distance of the order $5 \AA$, and all microscopic resonances are expected to be considerably detuned $(d \approx 1)$, at least in the outermost atomic layer. Then

$$
2 \pi a d / \lambda_{M}>10^{2} .
$$

From this we conclude that the condition (17) yielding $P^{0}=0$ is fulfilled unless the refractive index $\left(n_{1}\right.$ or $\left.n_{2}\right)$ is of the order 10 or larger. However, a detailed investigation of a typical resonance $\left(\epsilon_{\infty}<10\right.$ and $\left.F / \omega_{0}^{2}<1\right)$ indicates that modes with $|n|>10$ are negligibly excited whatever boundary condition is applied [case (a) or (b) above].

From these considerations, we see that it is reasonable to apply the condition $P=0$ at the surface throughout the spectrum for all dispersive resonances of practical interest.

It should be emphasized that our results strictly concern the long-wave limit. If the detuned layer has a width comparable to the reciprocal wave number $\left(1 / k_{1}\right.$ or $\left.1 / k_{2}\right)$ the boundary-condition problem is much more complicated. Such situations are also difficult in the absence of spatial dispersion since the optical properties of a surface change significantly if one introduces a surface layer with a polarizability different from that of the bulk and with a considerable optical retardation. Such surface layers associated with Wannier excitons have been investigated by Hopfield and Thomas. ${ }^{2}$

\section{SEVERAL RESONANCES}

So far we have only considered a single resonance. This treatment is easily generalized to include several dispersive resonances. Each extra resonance introduces an extra equation of motion for the contribution to the polarization. Hence, if there are $r$ resonances taken into account one obtains $r+1$ coupled equations analogous to Eqs. (2) and (3) which determine the propagation of the polariton waves. The dispersion of these waves is given by

$$
k^{2} c^{2} / \omega^{2}=\epsilon_{\infty}+\sum_{j=1}^{r} \frac{F_{j}}{\omega_{0 j}^{2}-\omega^{2}+\hbar \omega_{0 j} k^{2} / M_{J}} .
$$

This equation is of the order $r+1$ in $k^{2}$ and the $r+1$ roots determine the wave vectors of the eigenmodes. When setting up the boundary conditions we assume (as justified in Sec. IV) that all resonances are sufficiently detuned at the surface. Then we arrive at the boundary conditions given by the $r$ equations

$$
\sum_{i=1}^{r+1} P_{i, j}=0, \quad 1 \leqq j \leqq r
$$

at the surface. Here $P_{i, j}$ is the polarization contribution of the $j$ th resonance to the $i$ th polariton mode:

$$
P_{i, j}=\frac{-i \omega \epsilon_{0} F_{j} A_{i}}{\omega_{0 j}^{2}-\omega^{2}+\hbar \omega_{0 j} k_{i}{ }^{2} / M_{j}} .
$$

Hence, if more dispersive resonances are present in a material (20) requires that the contribution from each resonance to the polarization must vanish at the surface because of the detuning in the surface layer.

\section{COMPARISON WITH EXPERIMENTS}

Here we shall apply the theory outlined above to the $A$ - and $B$ - exciton lines in $\mathrm{ZnO}^{7}$ In order to establish clearly that the spatial-resonance dispersion effects are present in the $\mathrm{ZnO}$ low-temperature spectrum we shall concentrate on optical data which are less sensitive to damping, namely, the refractive index (measured by Fabry-Perot interference fringes) and the reflectivity. This is important since the exciton collision frequency may be so small that the mean free path becomes comparable to the sample thickness and to the magnitude of the reciprocal polariton wave vectors. The damping term may therefore contain contributions from complex nonlocal loss mechanisms such as exciton scattering at the surfaces. Hence the in- 

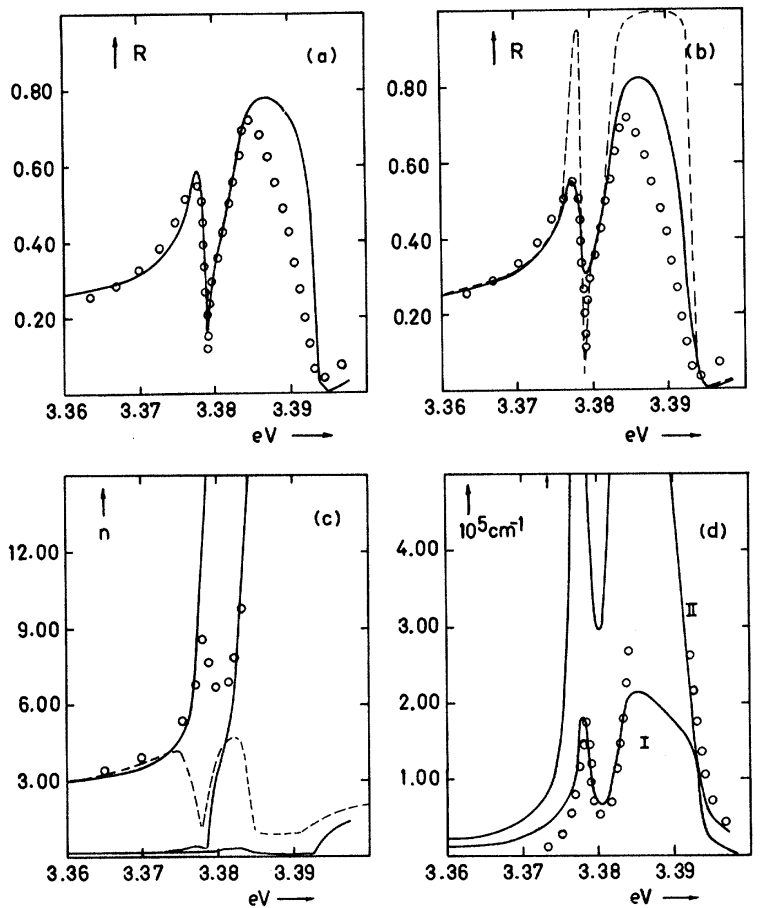

FIG. 3. Low-temperature exciton spectra of $\mathrm{ZnO}$. (a) Points indicate measured reflectivity (Ref. 9), while the curve is computed by including two dispersive resonances and applying the boundary condition of Eq. (22). The background dielectric constant was $\epsilon_{\infty}=6.2$ (Ref. 12) the exciton mass (equal for $A$ and $B$ ) $M=1.8 m_{0}$. The resonance parameters obtained from the fit were $\left(\hbar \omega_{T}\right)_{A}$ $=3.3772 \mathrm{eV},\left(\hbar \omega_{T}\right)_{B}=3.3828 \mathrm{eV}$ for the transverse resonance energies, and $f_{A}=2.6 \times 10^{-3}, f_{B}=5.2 \times 10^{-3}$ for the oscillator strengths per molecule. The reflectivity was rather independent of damping for $0<\Gamma<0.1 \mathrm{meV}$. Here we used $\Gamma=0.1 \mathrm{meV}$. (b) Attempts to fit reflectivity curves derived from two dispersive resonances and the boundary condition of Eq. (23) to the measured reflectivity The parameters used for the dashed curve are the same as in Fig. 3(a), while the damping was increased to $\Gamma=1.5$ $\mathrm{meV}$ for the other curve. (c) Points indicate measured refractive index (Ref. 8). The dashed curve is the result of a Kramers-Kronig analysis of the measured reflectivity [Fig. 3(a)]. Also shown are the refractive indices of the three polariton modes used in the calculation of Fig. 3(a). (d) Points indicate measured transmission (Ref. 15), given in the form $L^{-1} \log \left(T^{-1}\right)$, where $T$ is the transmission and $L$ the sample thickness, $L=2500 \AA$, I and II are transmission curves computed with parameters and boundary conditions as used in Figs. 3 (a) and 3 (b), respectively.

terpretation of absorption spectra is more troublesome.

First, we compare the Kramers-Kronig analysis of the measured reflectivity with the observed spectrum $^{8}$ of the refractive index. The result is shown in Fig. 3(c). It is seen that the observed refractive index deviates considerably from that derived classically from the reflectivity data. This indicates that the crystalline resonances responsible for the $A$ and $B$ lines are subject to spatial dispersion.

According to Thomas's assignment, ${ }^{7}$ both lines are due to free excitons. Estimates of the exciton mass of the $A$ and $B$ excitons in ZnO vary from $0.9 m_{0}$ to $2 m_{0}{ }^{10-12}$ Since we shall demonstrate the importance of spatial dispersion, we insert in our calculation the rather conservative value of $M=1.8$ $m_{0}$. Using this value we have fitted the $k=0$ resonance frequencies, the oscillator strengths, and a bulk (frequency-independent) damping to the measured reflectivity. We have used Eq. (19) with $r$ $=2$ and with the addition of a damping term equal to $2 i \Gamma \omega$ in the denominator on the right-hand side of Eq. (19). For simplicity we used the same value of $\Gamma$ for both resonances. The result of this $\mathrm{fit}^{13}$ is shown in Figs. 3(a) and 3(b) for the two kinds of boundary conditions

$$
\sum_{i=1}^{3} P_{i, j}=0, \quad j=1,2
$$

and

$$
\sum_{i=1}^{3} \frac{\partial P_{i, j}}{\partial x}=0, \quad j=1,2
$$

corresponding, respectively, to detuning (22) and perfect tuning (23) in the surface layer. It should be noted that the poor agreement in regions of high reflectivity is expected since minor irregularities of the surface tends to reduce the reflectivity in such regions. The fit in Fig. 3(a) (with the vanishing polarization at the surface) is rather insensitive to damping in the region $0 \leqq \Gamma<0.1 \mathrm{meV}$. As in Ref. 14 we find a satisfactory agreement between the calculated refractive index associated with the most dominant polariton mode and the experimental results [see Fig. 3(c)]. Furthermore, the oscillator strengths obtained in this fit are well explained in a previous paper. ${ }^{15}$

It has already been seen that the unlikely boundary condition of Eq. (23) is unable to reproduce the sharp reflectivity structure near the $A$ exciton. In order to demonstrate further the validity of the condition of Eq. (22) we have calculated the transmission of a slab applying the two extreme pairs of boundary conditions [Eqs. (22) and (23)] on both surfaces. In this case, the incoming wave will excite six polariton modes (three in each direction) and two waves outside the slab. Thus the total of eight boundary conditions provide the necessary equations. The results are shown in Fig. 3(d), where we have deliberately averaged out the interference fringes in both experimental and calculated spectra. Again the experiments favor the boundary condition predicted by theory [Eq. (22)]. It should be noted that the discrepancies still existing between calculated and observed transmission spectra might be ex- 
plained by incorporating nonlocal loss mechanisms.

\section{SUMMARY}

In this paper we have introduced a simple model which is believed to describe the relevant influence of a boundary on the polariton waves. In contrast to the considerations in Ref. 2, we do not neglect the coupling to light in the boundary-condition problem. Generally, our treatment leads to the condition that an appropriate linear combination of $P$ and $\partial P / \partial x$ vanishes at the surface [see Eq. (16)]. However, when inserting actual parameters of crys- talline resonances we find that $P=0$ at the surface is an adequate condition throughout the spectrum. The influence of the thickness $a$ of the surface layer has been treated strictly in the long-wave limit. Thus, if a polariton mode carrying a considerable part of the energy has a wave vector comparable to $1 / a$, then a more elaborate calculation is necessary. ${ }^{2}$ We have also extended the theory of bulk waves and boundary conditions to include two or more dispersive resonances. Finally, we have demonstrated the agreement with experiment of the boundary condition in case of the exciton lines in $\mathrm{ZnO}$.
${ }^{1}$ S. I. Pekar, Zh. Eksperim。i Teor. Fiz. 33, 1022 (1957) [Sov. Phys. JETP $\underline{6}, 785$ (1958)].

${ }^{2}$ J. J. Hopfield and D. G. Thomas, Phys. Rev. 132, 563 (1963).

${ }^{3}$ J. J. Hopfield, J. Phys. Soc. Japan, Suppl. 21, 777 (1966).

${ }^{4}$ V. M. Agranovich and V. L. Ginzburg, Spatial Dispersion in Crystal Optics and the Theory of Excitons (Interscience, John Wiley and Sons, London, 1966).

${ }^{5}$ C. W. Deutsche and C. A. Mead, Phys. Rev。 158, A63 (1965).

${ }^{6}$ J. J. Hopfield, Phys. Rev. 112, 1555 (1958).

${ }^{7}$ D. G. Thomas, J. Phys. Chem. Solids $\underline{15}, 86(1960)$.

${ }^{8}$ Y. S. Park and J. R. Schneider, J. Appl. Phys. 39, 3049 (1968)

${ }^{9} \mathrm{~J}$. Filinski and T. Skettrup (unpublished).
${ }^{10}$ M. Cardona, J. Phys. Chem. Solids 24, 1543 (1963) $\left(M=0.9 m_{0}\right)$.

${ }^{11}$ D. E. Dietz, J. J. Hopfield, and D. G. Thomas, J. Appl. Phys。32 Suppl. 2282 (1961). $\left(M=1.5 m_{0}\right.$, if corrected for the new value of the electron mass, $\left.m_{e}=0.25 m_{0}\right)$.

${ }^{12}$ B. Segall, Phys. Rev. $\underline{163}, 769$ (1967) $\left(M=2 m_{0}\right)$.

${ }^{13}$ The time-saving approximations introduced in previous computations of exciton spectra in $\mathrm{ZnO}$ (see Ref. 13) have not been applied here. Consequently, we obtain somewhat different values for oscillator strengths and resonance frequencies.

${ }^{14} \mathrm{~T}$. Skettrup, Solid State Commun. 7, 869 (1969).

${ }^{15} \mathrm{~T}$. Skettrup and I。Balslev, Phys. Stat. Solidi $\underline{40}$, 93 (1970).

\title{
Focusing of Phonons in Crystalline Solids due to Elastic Anisotropy*
}

\author{
B. Taylor, H. J. Maris, and C. Elbaum \\ Department of Physics, Brown University, Providence, Rhode Island 02912 \\ (Received 14 September 1970)
}

\begin{abstract}
Large differences (up to a factor of 100) have been observed in the intensity of phonons of different polarizations propagating ballistically in $\mathrm{LiF}, \mathrm{KCl}$, and $\mathrm{Al}_{2} \mathrm{O}_{3}$. These observations were made by means of heat-pulse experiments carried out on single crystals, in the temperature range $1.5-3.5^{\circ} \mathrm{K}$. The results are explained in terms of phonon focusing due to the fact that in elastically anisotropic crystals the phonon phase and group velocities a re, in general, not collinear. Calculations of the focusing effect, to determine the relative intensities of phonons of each polarization in many crystal directions, have been carried out for a number of solids. The results of these calculations are in good agreement with the experimental results obtained in this study, as well as with those obtained by other investigators on Si, Ge, and NaF.
\end{abstract}

\section{INTRODUCTION}

In heat-pulse experiments, ${ }^{1}$ phonons are generated in a thin metallic film on one face of a crystal by means of short electrical, microwave, or laser pulses. The phonons are detected, after propagating through the crystal, by means of a suitable bolometer. Metallic films which undergo a superconducting-to-normal transition when the phonon energy arrives are very effective detectors. For heat-pulse experiments, a crystal with phonon mean free path comparable to its linear dimensions is required so that the phonons will propagate ballisti cally rather than diffusively. Pulses due to phonons of different polarizations then arrive at the detector at different times and therefore can be studied independently. These experiments have considerable advantages over thermal-conductivity studies 